\title{
BMJ Open Investigating Canadian parents' HPV vaccine knowledge, attitudes and behaviour: a study protocol for a longitudinal national online survey
}

\author{
Gilla K Shapiro, ${ }^{1,2}$ Samara Perez, ${ }^{1,2}$ Anila Naz, ${ }^{2}$ Ovidiu Tatar, ${ }^{2}$ Juliet R Guichon, ${ }^{3}$ \\ Rhonda Amsel, ${ }^{1}$ Gregory D Zimet, ${ }^{4}$ Zeev Rosberger ${ }^{1,7,2,5,6}$
}

To cite: Shapiro GK, Perez S, $\mathrm{Naz} A$, et al. Investigating Canadian parents' HPV vaccine knowledge, attitudes and behaviour: a study protocol for a longitudinal national online survey. BMJ Open 2017;7:e017814. doi:10.1136/ bmjopen-2017-017814

- Prepublication history and additional material for this paper are available online. To view these files, please visit the journal online (http://dx.doi org/10.1136/bmjopen-2017017814).

Received 18 May 2017 Revised 27 July 2017 Accepted 1 August 2017

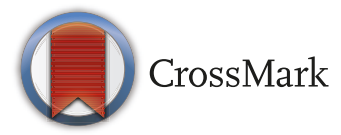

For numbered affiliations see end of article.

Correspondence to

Gilla K Shapiro;

gilla.shapiro@mail.mcgill.ca

\section{ABSTRACT}

Introduction Human papillomavirus (HPV), a sexually transmitted infection, can cause anogenital warts and a number of cancers. To prevent morbidity and mortality, three vaccines have been licensed and are recommended by Canada's National Advisory Committee on Immunisation (for girls since 2007 and boys since 2012). Nevertheless, HPV vaccine coverage in Canada remains suboptimal in many regions. This study will be the first to concurrently examine the correlates of HPV vaccine decision-making in parents of school-aged girls and boys and evaluate changes in parental knowledge, attitudes and behaviours over time.

Methods and analysis Using a national, online survey utilising theoretically driven constructs and validated measures, this study will identify HPV vaccine coverage rates and correlates of vaccine decision-making in Canada at two time points (August-September 2016 and June-July 2017). 4606 participants will be recruited to participate in an online survey through a market research and polling firm using email invitations. Data cleaning methods will identify inattentive or unmotivated participants.

Ethics and dissemination The study received research ethics board approval from the Research Review Office, Integrated Health and Social Services University Network for West-Central Montreal (CODIM-FLP-16-219). The study will adopt a multimodal approach to disseminate the study's findings to researchers, clinicians, cancer and immunisation organisations and the public in Canada and internationally.

\section{INTRODUCTION}

Human papillomavirus (HPV) is the most common sexually transmitted infection. ${ }^{1-4}$ While most HPV infections are asymptomatic and do not progress to disease ${ }^{5-7}$ some infections can cause substantial morbidity and mortality. ${ }^{8-11}$ It is estimated that $5.2 \%$ of all worldwide cancers are attributable to HPV. ${ }^{11-13}$ HPV-associated cancers include cervical, oropharyngeal, anal, vaginal, vulvar and penile. ${ }^{911}$ Combined, HPV is responsible for over 4000 new cancer cases annually in

\section{Strengths and limitations of this study}

- Strengths of the study include a large sample size $(n=4606)$, a nationally representative sample, use of psychometrically validated scales, the use of theoretical frameworks, a mixed methods approach, a wider range of constructs than in previous studies and sophisticated data cleaning techniques to exclude inattentive or unmotivated responders.

- Limitations of this study include relying on selfreported data.

Canada. ${ }^{9}$ Certain strains of HPV (eg, HPV $6 / 11$ ) also cause anogenital warts. ${ }^{14}$ HPV-associated disease can impact quality of life and accrue substantial costs to the healthcare system. 91015

Three prophylactic vaccines that prevent against the oncogenic strains of HPV have been developed and recommended: the bivalent Cervarix, quadrivalent Gardasil and nonavalent Gardasil. ${ }^{9} 16$ These vaccines are safe and effective. ${ }^{17-23}$ Canada's National Advisory Committee on Immunisation (NACI) has recommended the HPV vaccines for girls (since 2007) and boys (since 2012) ages 9-26. ${ }^{2} 16$ From 2007 to 2010, all Canadian provinces and territories implemented publicly funded, school-based vaccination programmes for girls, although at different ages (ie, 9-13 years of age) and with different dosing schedules (ie, two or three doses). ${ }^{24} 25$ Vaccinating children at this age provides the highest level of immunogenicity and protects individuals before they are sexually active and thereby at risk of infection. ${ }^{920}$ This approach is similar to the majority of countries that provide publicly funded HPV vaccination programmes to girls. ${ }^{26}$

To date, only a handful of countries have extended their publicly funded, school-based HPV vaccination programmes to boys. ${ }^{25} 2728$ 


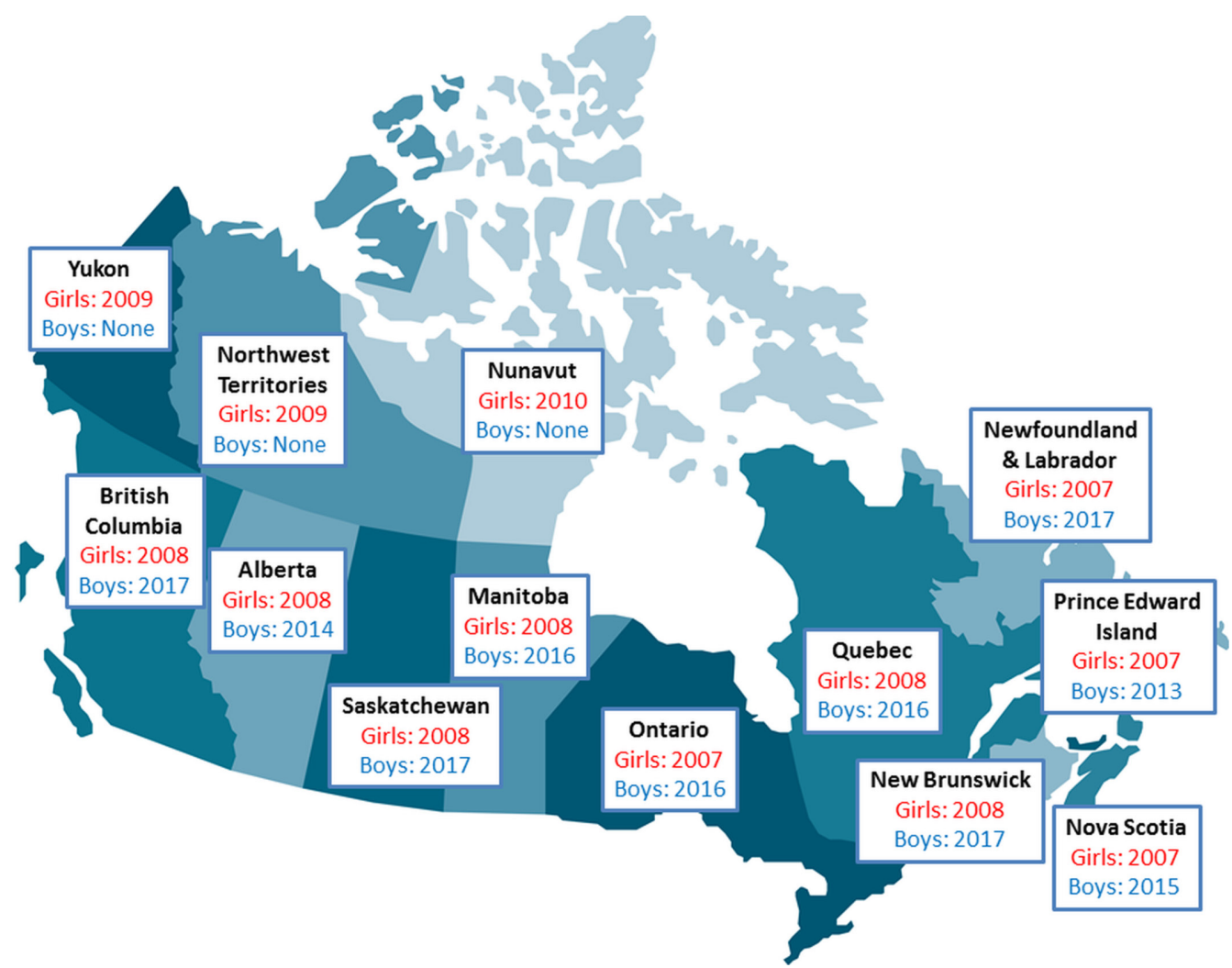

Figure 1 Publicly funded school-based HPV vaccine programmes in Canada. This figure identifies the year that publicly funded school-based HPV vaccine programmes were initiated for girls and boys by Canadian jurisdiction.

Canada has been an international leader in providing gender-neutral HPV vaccination ${ }^{25}$ by September 2017 , 10 of Canada's 13 regions will have commenced schoolbased HPV vaccination programmes that include boys (figure 1). ${ }^{29-36}$ However, implementation of male HPV vaccination across Canada has been staggered, presenting a natural experiment to evaluate and compare the impact of the introduction of the HPV vaccine programmes on parents' attitudes, knowledge and vaccine coverage.

Achieving high levels of vaccine coverage protects individuals and helps prevent transmission to unvaccinated partners, which maximises population-level effectiveness (ie, through herd protection). ${ }^{37} \mathrm{HPV}$ vaccination programmes in Canada are not reaching their target rates of immunisation. ${ }^{38}$ HPV vaccine uptake rates in Canada vary considerably by region; in a national survey of parents of girls aged 12-14years, Gilbert et al reported vaccination rates between $52.6 \%$ and $89.7 \%$ (2013 data). ${ }^{40}$ On average, HPV vaccine uptake across Canada was $72.3 \% .^{40}$ Preliminary evidence for boys in PEI's schoolbased vaccination programme indicates $85.4 \%$ vaccine uptake (2013/2014 data). ${ }^{29}$ However, a national survey of Canadian parents found uptake rates for boys in the context of (only one then two) publicly funded schoolbased programmes was extremely low $(<3 \%$ nationwide; 2013 data). ${ }^{41}$ The lack of a national immunisation registry makes it difficult to compare HPV vaccine coverage rates, and no national survey has yet examined HPV vaccine coverage in girls and boys simultaneously.
Given that parental consent is required for schoolbased immunisation programmes for children in Canada, the NACI and Canadian Immunisation Committee (CIC) have made it a research priority to understand why parents delay or refuse to vaccinate their children. ${ }^{9}{ }^{24}$ Accordingly, this study seeks to understand the sociodemographic, psychosocial and behavioural correlates of HPV vaccine coverage. Over the last decade, a number of studies have identified factors associated with HPV vaccination decision-making including demographics, knowledge, attitudes, social norms, logistics (eg, time, effort) and cost. ${ }^{42-53}$ The evidence has indicated some common themes (eg, the importance of physician recommendation, perceived benefit, perceived safety, cost), and some contradictory evidence (eg, knowledge has been found to correlate both negatively and positively with vaccine acceptance). ${ }^{48} 4954$ The degree to which each of these factors contributes (ie, the effect size) and possible policy variations between jurisdictions remains largely unclear. In addition, despite several systematic reviews, ${ }^{48} 49515355$ not all potentially relevant factors (eg, the effect of vaccine conspiracy beliefs) have been identified or comprehensively investigated in large population-based studies, especially in the Canadian context. ${ }^{56}$ Furthermore, the majority of studies addressing parental HPV vaccine decision-making have been primarily focused on parents of girls, ${ }^{40} 57$ with fewer studies evaluating and making comparisons with parental HPV vaccine decision-making for boys. ${ }^{58-60}$ 
The present study aims to address these research gaps. Using a national, online survey utilising theoretically driven constructs and validated questionnaires, this study identifies HPV vaccine coverage and correlates of decision-making in Canada. It will be the first to study concurrently the correlates of decision-making in Canadian parents of eligible school-aged girls and boys. This study will administer a survey at two time points (AugustSeptember, 2016 and June-July, 2017) to capture important factors related to HPV vaccine hesitancy, acceptance and variation over time. Accordingly, this study will elucidate psychosocial factors that influence parents to vaccinate their daughters or sons contemporaneously and evaluate changes in parental knowledge, attitudes and behaviours over time.

\section{Study objectives and hypotheses}

The main objectives of this study are as follows:

1. To describe HPV vaccine coverage in Canadian girls and boys

In the absence of a national immunisation registry, ${ }^{61}$ current information on HPV vaccine coverage is unclear and continually evolving. We aim to determine HPV vaccine coverage in girls and boys nationally and across Canadian jurisdiction, and how rates change over time.

2. To assess the correlates of HPV vaccination in parents of girls and boys

In order to improve programmes, it is important to understand the factors associated with HPV vaccine uptake. To date, Canadian studies have assessed the correlates of HPV vaccine uptake in parents of girls and parents of boys separately. ${ }^{52}$ Because the HPV vaccine is available to girls and boys of varying ages, we aim to understand the determinants of HPV vaccine uptake in these groups, using constructs from the Health Belief Model (HBM), a commonly used theoretical model that includes core beliefs that are hypothesised to predict the adoption of new health behaviours. ${ }^{62}$ The HBM has been used to examine various health-related behaviours, including cancer prevention and vaccination. ${ }^{63}$ As applied to HPV vaccination, elements of the HBM include perceived benefits of, and barriers to, HPV vaccination; perceived severity of, and susceptibility to, HPV infection and disease and external influences prompting HPV vaccine uptake (ie, cues to action). This study will use HBM constructs and other important predictors to evaluate, compare and contrast. We hypothesise that higher HPV vaccine uptake will be significantly related to greater HPV knowledge, HBM constructs (particularly lower 'barriers' and more 'cues to action'), non-HBM attitudinal constructs (eg, lower vaccine conspiracy beliefs) and healthcare provider (HCP) recommendation. In parents of boys, a publicly funded programme (that reduces barriers of cost and access) and HCP recommendation are hypothesised to be particularly important.
3. To identify Canadian parents' stage of decision-making by gender and province

Few studies have examined the stages of parents' vaccine decision. Assessing differences in parents' HPV vaccine decision-making stage is important for identifying how best to intervene for parents at different stages. ${ }^{58} 65$ Using the Precaution Adoption Process Model (PAPM), a stage-based theoretical model, we will classify parents according to their unique stage of HPV vaccine decision-making and examine the associated attitudes with that stage ${ }^{65-67}$ The PAPM, as applied to HPV vaccination, identifies individuals along six stages of decision-making: (1) unaware of the vaccine, (2) unengaged in the decision to vaccinate their child, (3) undecided about whether to vaccinate their child, (4) decided not to act (ie, decided not to vaccinate their child), (5) decided to act (ie, decided to vaccinate their child) and (6) acted (ie, vaccinated their child) (see online supplementary material 1 ). We will compare the stage of decision-making of parents of girls with boys, as well as the stages of parents in those regions that have publicly funded programmes for boys at Time 1 (ie, Alberta, Nova Scotia, PEI) with those that do not (ie, British Columbia, Manitoba, New Brunswick, Newfoundland and Labrador, Northwest Territories, Nunavut, Ontario, Saskatchewan, Quebec, Yukon). We hypothesise that parents of boys will be in earlier PAPM stages compared with parents of girls and that parents of boys in regions without an HPV vaccine programme will be in earlier stages compared with parents of boys in regions with a programme.

4. To determine the impact of publicly funded HPV vaccine programme initiation for boys

HPV vaccination programmes for boys were implemented in Quebec, Ontario and Manitoba in the Fall of 2016, which is after Time 1 survey administration (August-September 2016), but before launching the Time 2 survey (June-July 2017). This natural experiment allows us to evaluate the impact of introducing a public school-based HPV vaccination programme on parents' HPV vaccine knowledge, attitudes and HPV vaccine coverage. Accordingly, we will assess whether these factors change from Time 1 to Time 2. We hypothesise that compared with programmes with no change to their public vaccination programme, at Time 2 (post-intervention) parents of boys in Manitoba, Ontario and Quebec will have increased HPV vaccine knowledge, more positive HPV vaccine attitudes, be more likely to have received a HCP's recommendation and be more likely to have received the HPV vaccine. British Columbia, Saskatchewan, New Brunswick and Newfoundland and Labrador have announced that they would fund HPV vaccine for boys to begin in September 2017 (figure 1); this research will therefore have value in predicting how parental attitudes regarding vaccinating their sons might change in those jurisdictions. 


\section{METHODS AND ANALYSIS}

\section{Study design}

This study uses a longitudinal design to collect self-reported data through an online questionnaire from a large national sample of Canadian parents. Surveys are administered at two time points: Time 1 during AugustSeptember (2016) and Time 2 during June-July (2017). Participants who responded to the survey at Time 1 were contacted again at Time 2 using the same questionnaire.

\section{Sample}

This study targets parents and guardians (hereafter referred to as 'parents') of 9 to 16 year old girls and boys across Canada. This population is targeted because, on the younger side (ie, age 9), it includes the youngest children included in NACI's recommendation and, on the older side (ie, age 16), it includes children who, generally speaking, require parental consent in Canada. ${ }^{68}$ Parents will be recruited by Canada's largest market research and polling firm, Leger-The Research Intelligence Group. Leger maintains a national panel of 400000 Canadians who have internet access, reside in Canada and are fluent in English or French. This study targeted parents who have a child between 9 and 16 years of age living in their household. Participants completing the questionnaire at Time 1 will be contacted again at Time 2 .

Leger's panels include individuals of all profiles with regard to gender, age, education level, household composition and income for all regions, making it feasible to target specific participants effectively. ${ }^{67}$ The panel is constructed to be nationally, as well as regionally, representative. Leger uses proprietary software informed by Canada's census data in order to generate a representative sample of the population. Leger's software follows an interactive algorithm to invite participants according to specified eligibility criteria. In this study, Leger's software enables extraction of all active and available panellists who meet the screening criteria, random sorting of the selected sample pool, examination of the number of panellists who satisfy each target group (ie, parents of girls aged 9-16year or parents of boys aged 9-16year) and recalculation and balancing of the sample across the target groups. To recruit participants, Leger sends an email invitation and survey link to selected panellists. Leger sends a maximum of three reminder emails to its selected panellists to complete the survey until the required numbers of participants are recruited.

This study's sample size calculation takes into account previous research indicating that approximately $15 \%$ of respondents are inattentive or unmotivated responders who would be excluded when using rigorous data cleaning methods. ${ }^{66}{ }^{69}$ To evaluate different stages of decision-making (objective 3), we are guided by previous research that found few individuals in particular stages (especially in less populated regions) ${ }^{66}$ An attrition rate (of approximately $40 \%-50 \%$ ) from the first wave of data collection (Time 1, August-September 2016) to the second wave (Time 2, June-July 2017) is also expected. ${ }^{66}$
Therefore, in order to attain a sufficient number of respondents to enable analyses of HPV vaccine decision-making by stage and region, this study recruits approximately 4600 parents of school-aged children (ages 9-16) at Time 1 , equally divided between parents of girls and parents of boys.

\section{Measures}

We use an online questionnaire that incorporates intelligent programming, such that the child's first name is included in the survey questions and parents receive questions that are personalised for them. Questionnaire items include previously validated scales. ${ }^{70-74}$ Participants will be asked to identify themselves as a parent or guardian, report the number of children they have and their children's ages and genders. Parents with more than one child who meet the inclusion criteria will be asked to answer the questionnaire for the child who has had the most recent birthday, a randomisation technique previously employed ${ }^{66}$ The questionnaire assesses sociodemographics, HPV and HPV vaccine knowledge (using validated scales), ${ }^{71}$ PAPM stage, HPV vaccine willingness, HPV vaccine coverage, HCP recommendation (including the strength of the recommendation), HPV attitudes (using validated scales), ${ }^{73}$ motivation towards vaccination, vaccine hesitancy (using a developed scale) ${ }^{74}$ and vaccine conspiracy beliefs (using a validated scale). ${ }^{70}$ Items within validated questionnaires are administered in a random order to ameliorate any order effect and invariant responding. ${ }^{76}$ Five open-ended qualitative questions will provide nuance in capturing details of parents' subjective perspectives on decision-making. ${ }^{77}$ A detailed description of the questionnaire's items can be found in the online supplementary material 1 .

To take into account language and literacy level, the questionnaire was adjusted to a grade eight reading level. To ensure the questionnaire could be answered in either of Canada's national languages, the English questionnaire was translated into French using Asiatis, an international translation service company. Bilingual team members verified the French translation and back translation.

\section{Data collection and management}

Leger will facilitate data collection. Participants will be sent an email invitation to participate in a questionnaire and then assigned a unique access number. By accessing the questionnaire with this unique number, the respondent enters a secure account that ensures confidentiality. Moreover, if necessary, respondents may stop and resume the questionnaire where they left off so that they participate at a time that best suits them, allowing them to complete the questionnaire conscientiously.

Participants will be paid a modest cash amount in accordance with standard panel member compensation of Leger. Data collection at both time periods will be completed within fourweeks. Missing data will not be an obstacle in this survey because participants will be required to answer all questions before moving from one 
page to the next. Once participants complete the questionnaire, they will be debriefed, informed about HPV vaccination and provided with informational resources. Leger will transfer the anonymised raw data file to our research team, which will be stored on a secure server at the Lady Davis Institute for Medical Research site of the Integrated Health and Social Services University Network for West-Central Montreal.

\section{Data cleaning procedure}

As recommended in the literature ${ }^{69} 7678$ data cleaning methods will be used to identify participants who might not have used appropriate care while completing the questionnaire (ie, inattentive or unmotivated responders). Consistent with DeSimone et al (2015) and Perez et al (2016), we will use data cleaning methods that are direct (ie, bogus items) and statistical (ie, psychometric synonyms and psychometric antonyms). ${ }^{6676}$

Bogus items will be used to screen inattentive or unmotivated responders. Two bogus items on Likert scales were randomly inserted into the survey: 'I have never met anyone younger than I am' and 'I have been to every country in the world' (measured from ' 1 -strongly disagree' to '7—strongly agree'). Incorrect answers (ie, agreement) to both bogus items suggest inattentive or unmotivated responders. Incorrect responses are indicative of lack of attention. Respondents who answer at least one bogus item correctly will be retained.

Psychometric synonyms and antonyms data cleaning methods statistically examine response patterns. ${ }^{66}$ Providing different responses to similar items suggests insufficient attention and accuracy in answering the questionnaire. Items measured on Likert scales will be selected and inter-item correlations will be calculated. Positively correlated pairs of items will constitute psychometric synonyms while negatively correlated pairs will constitute psychometric antonyms. ${ }^{76}$ The number of pairs cannot be anticipated before beginning data analysis because it depends on the degree of correlation and the chosen cut-off value of the correlation coefficient. ${ }^{76}$ We will use an inter-item Pearson correlation cut-off of 0.60 and -0.60 for selecting psychometric synonyms and psychometric antonyms pairs, respectively, consistent with recommendations of Meade and Craig (2012) ${ }^{78}$ Once the pairs have been identified, an index will be calculated for each respondent by correlating the responses to the first items of the pairs with the responses to the second items of the pairs.

Responders who meet both the bogus item and the psychometric synonyms/antonyms criteria will be considered attentive responders and retained.

\section{Data analysis plan}

In line with the first research objective to provide an accurate description of HPV vaccine coverage, we will report $\mathrm{HPV}$ vaccination coverage as percentage of girls and boys in each region and age group whose parents report they have received one, two or three doses of the HPV vaccine.
To test for statistically significant differences in proportions, we will use Pearson's $\mathrm{X}^{2}$ square tests and two sample tests of proportions.

For the second objective, to assess the correlates of HPV vaccine uptake in Canada, HPV vaccine uptake (dependent variable) will be dichotomised into 'vaccinated' (ie, received at least one dose of the HPV vaccine) and 'non-vaccinated'. Logistic regressions will be used to estimate the odds of vaccine uptake based on the correlates of interest, including sociodemographics, attitudes (informed by the HBM), knowledge and behaviours (eg, discussion with HCP). Significant associations between the correlates and vaccine uptake will be tested using bivariate logistic regression analyses. Multivariate logistic regression modelling will then be performed with all correlates from the bivariate analysis entered simultaneously. The model will be tested for goodness of fit, discrimination capacity and multicollinearity.

To identify Canadian parents' stage of decision-making by gender and region (objective 3), we will report parents' HPV vaccine decision-making in percentages based on the six stages of the PAPM. For assessing significant differences in PAPM stage based on gender (at Time 1) and availability of publicly funded HPV vaccination programmes for boys (at both Time 1 and Time 2), Pearson $\mathrm{X}^{2}$ test and two sample tests of proportions will be used.

Lastly, to determine the impact of publicly funded HPV vaccine programme initiation for boys in some regions, we will examine changes in parents' of boys (HPV and HPV vaccine) knowledge and attitudes (eg, on the HPV Attitudes and Beliefs Scale, Vaccine Conspiracy Beliefs Scale and Vaccine Hesitancy Scale) before and after the introduction of the funded programme. Parents of boys in provinces that introduced the programme will be compared with parents of boys in regions with no change to their programme. Significant differences from Time 1 to Time 2 will be tested using paired t-tests.

\section{ETHICS AND DISSEMINATION \\ Study ethics}

The study received research ethics board approval from the Research Review Office, Integrated Health and Social Services University Network for West-Central Montreal (CODIM-FLP-16-219). This is a university-affiliated teaching healthcare network where the coordinating centre (Lady Davis Institute for Medical Research) is based. Study participants consented to Leger's terms of use and privacy policy, which indicates that their data will be used anonymously for the research study.

\section{Dissemination plan}

The study will adopt a multimodal approach to disseminate the study's results to researchers, clinicians, cancer and vaccination organisations and the public in Canada and internationally. Study findings will be published in peer-reviewed scientific journals (including open 
source). To assure wide availability of our results to the research community, journals will be selected that reach both research and health professional audiences.

Presentations will be made at national and international scientific meetings and symposia, such as the Canadian Immunisation Conference, Canadian Association of Psychosocial Oncology, International Papillomavirus Conference and the International Psycho-Oncology Society. In addition, we will share the results with NACI, CIC and provincial immunisation advisory boards.

Given that the data is timely and could have immediate, direct implications for public education of Canadian parents and more widespread influence on public health policy, we will prioritise analysis and dissemination of projects that have a potential for proximal public impact. We hope that sharing outcomes with non-profit organisations (eg, the Canadian Cancer Society) will provide important platforms for innovative educational interventions based on this study's findings.

We will draft lay research summaries in media releases for dissemination to national media outlets to help the public understand the importance of this research. Media releases will also be used to bring the issues and challenges related to HPV vaccine acceptance to the public domain in order to inform discussions about HPV vaccination.

\section{DISCUSSION}

\section{Study implications}

By surveying a population-based representative sample of parents of eligible children, this study will provide current information about HPV vaccine coverage rates for both girls and boys nationally and across Canadian jurisdictions (objective 1). Since HPV vaccine programmes and policies are constantly evolving, ${ }^{25}$ it is timely to evaluate comprehensively variations in programme outcomes that target girls and boys, jurisdictions at a national and local level and HPV vaccination by sociodemographic groups. For this reason, this study will be useful to policymakers in understanding where the HPV vaccination programmes are meeting coverage targets, where disparities in vaccination exist, and which groups or jurisdictions may benefit from interventions designed to increase vaccination.

In order to improve the impact of publicly funded HPV vaccination programmes, this study will examine the psychosocial and behavioural factors associated with parents' decisions to vaccinate their children and their decision-making stage (objectives 2 and 3). These theoretically driven investigations will enable policymakers to develop interventions to increase HPV vaccination that are evidence-based, tailored and targeted towards parents' unique informational needs and their stage of decision-making, rather than providing all parents with the same messages.

Lastly, since Canada is one of the few countries that have implemented publicly funded, national HPV vaccination programmes, this research will make use of a natural experiment to evaluate the impact of the introduction of funded programmes for boys on parents' vaccine knowledge, attitudes and decision to vaccinate (objective 4). The results of this study will improve our understanding of the complex interplay of psychosocial and behavioural factors with policy decisions. By understanding this complexity, other countries can better anticipate the impact of policy changes on vaccination.

The results generated by the study's four objectives will provide public health officials with critical information about HPV vaccination programmes, improve the field's understanding of influencers of decision-making, enhance the delivery of publicly funded HPV vaccination programmes, facilitate HPV vaccine uptake and in turn decrease Canada's cancer burden and the associated human and economic cost.

\section{Methodological strengths}

The recruitment strategy of using a marketing company (Leger) that maintains a nationally representative panel for data collection enables the sampling of a large number of parents who answer the survey within a short time frame. The precise recruitment period allows for data collection to occur in a timely manner and the provision of a snapshot of responses before and after the implementation of the HPV vaccination programme for boys in certain provinces.

In addition, we use an online survey methodology with intelligent programming, which increases the quality of collected data by personalising and tailoring the survey for each participant. This study also avoids the problems associated with missing data. To avoid the limitation of inattentive or unmotivated responding that is often found in survey data, this study will utilise sophisticated data cleaning techniques to remove such responders.

Further, the measures used in this study include psychometrically validated scales (where possible), which increase the reliability and validity of our results. Our survey also assesses diverse constructs. By using theoretical frameworks (such as the HBM and PAPM), we will be able to better understand the vaccine acceptability process, which is important in nuanced targeting of interventions. Lastly, by including quantitative and qualitative (open-ended) questions, we will be able to conduct additional mixed-methods analyses to examine in-depth explanations of HPV vaccine decision-making at different stage levels.

\section{Foreseeable limitations}

One limitation of this study is the reliance on parents' self-reports of vaccination status for their children. In order to minimise this limitation, parents are asked about their child's vaccination status before and after reading an informative statement (whereby parents are provided details about the HPV vaccine). The exact number of reported doses (two or three) could also be inaccurate. A possible way to confirm immunisation status is to request parents to check the immunisation record, contact the family doctor or link to provincial immunisation records. 
However, requesting participants to access records is not feasible in our study as such a request would significantly increase the data collection time and costs. Another limitation of this study's design is that because we assess the same population at two time points, this study does not control for knowledge changes that occur as a result of the first survey. As our study's objective (objective 4) is to compare provinces with and without provincial funding, this study makes the assumption that knowledge changes as a result of the first survey affects individuals from all provinces equally.

\section{Author affiliations}

${ }^{1}$ Department of Psychology, McGill University, Montreal, Quebec, Canada

${ }^{2}$ Lady Davis Institute for Medical Research, Jewish General Hospital, Montreal, Quebec, Canada

${ }^{3}$ Department of Community Health Sciences, Faculty of Medicine, University of Calgary, Calgary, Alberta, Canada

${ }^{4}$ Section of Adolescent Medicine, Department of Pediatrics, Indiana University School of Medicine, Indianapolis, Indiana, USA

${ }^{5}$ Louise Granofsky Psychosocial Oncology Program, Segal Cancer Center, Jewish General Hospital, Montreal, Quebec, Canada

${ }^{6}$ Department of Psychiatry, McGill University, Montreal, Quebec, Canada

${ }^{7}$ Department of Oncology, McGill University, Montreal, Quebec, Canada

Acknowledgements The authors appreciate the guidance and expertise of Dr Eve Dubé, Dr Vladimir Gilca, Professor Gina Ogilvie and Professor Eduardo Franco.

Contributors GKS conceived and designed the study, developed the survey and wrote the manuscript. AN and OT participated in designing the study, assisted in drafting the manuscript and provided critical feedback on manuscript revisions. SP, JRG, GDZ and RA provided critical feedback on manuscript revisions. ZR conceived and designed the study, developed the survey and provided critical feedback on manuscript revisions. All authors read and approved the final manuscript.

Funding This work was supported by the Canadian Cancer Society Research Institute under the title 'Understanding HPV Vaccine Hesitancy among Canadian Parents' (grant number: 704036). GKS was supported by the Vanier Canada Graduate Scholarship and Queen Elizabeth II Diamond Jubilee Scholarship programs.

Competing interests ZR reports grants from the Canadian Cancer Society Research Institute during the conduct of the study and personal fees from Merck outside the submitted work. GDZ reports grants from Merck and personal fees from Sanofi Pasteur, outside the submitted work. The remaining authors declare no conflict of interest.

Ethics approval The study received research ethics board approval from the Research Review Office, Integrated Health and Social Services University Network for West-Central Montreal (CODIM-FLP-16-219).

Provenance and peer review Not commissioned; externally peer reviewed.

Open Access This is an Open Access article distributed in accordance with the Creative Commons Attribution Non Commercial (CC BY-NC 4.0) license, which permits others to distribute, remix, adapt, build upon this work non-commercially, and license their derivative works on different terms, provided the original work is properly cited and the use is non-commercial. See: http://creativecommons.org/ licenses/by-nc/4.0/

(c) Article author(s) (or their employer(s) unless otherwise stated in the text of the article) 2017. All rights reserved. No commercial use is permitted unless otherwise expressly granted.

\section{REFERENCES}

1. Shearer BD. HPV vaccination: understanding the impact on HPV disease. 34: Purple Paper, 2011. http://www.nccid.ca/files/Purple_ Paper_Note_mauve/PP_34_EN.pdf

2. National Advisory Committee on Immunization. Update on the recommended human papillomavirus vaccine immunization schedule. 2015 http://publications.gc.ca/collections/collection_2015/ aspc-phac/HP40-128-2014-eng.pdf (accessed 08 May 2017).
3. Crosbie EJ, Einstein MH, Franceschi S, et al. Human papillomavirus and cervical cancer. Lancet 2013;382:889-99.

4. Moore RA, Ogilvie G, Fornika D, et al. Prevalence and type distribution of human papillomavirus in 5,000 British Columbia women -implications for vaccination. Cancer Causes Control 2009;20:1387-96.

5. Little KQ, Ogilvie G, Mirwaldt P. Human papillomavirus awareness, knowledge, and vaccination status in a diverse population of male postsecondary students in Greater Vancouver. BC Med J 2015;57.

6. Parkin DM, Bray F. Chapter 2: the burden of HPV-related cancers. Vaccine 2006;24:S11-S25.

7. Steben M, Duarte-Franco E. Human papillomavirus infection: epidemiology and pathophysiology. Gynecol Oncol 2007;107:S2-S5.

8. Bouvard V, Baan R, Straif K, et al. A review of human carcinogens part B: biological agents. Lancet Oncol 2009;10:321-2.

9. National Advisory Committee on Immunization. Update on human papillomavirus vaccines. 38: Canada Communicable Disease Report, 2012.

10. Forman D, de Martel C, Lacey CJ, et al. Global burden of human papillomavirus and related diseases. Vaccine 2012;30(Suppl 5):F12-F23

11. de Martel C, Ferlay J, Franceschi S, et al. Global burden of cancers attributable to infections in 2008: a review and synthetic analysis. Lancet Oncol 2012;13:607-15.

12. Parkin DM. The global health burden of infection-associated cancers in the year 2002. Int J Cancer 2006;118:3030-44.

13. Oh JK, Weiderpass $\mathrm{E}$. Infection and cancer: global distribution and burden of diseases. Ann Glob Health 2014:80:384-92.

14. Ball SL, Winder DM, Vaughan K, et al. Analyses of human papillomavirus genotypes and viral loads in anogenital warts. J Med Virol 2011;83:1345-50.

15. Marra F, Ogilvie G, Colley L, et al. Epidemiology and costs associated with genital warts in Canada. Sex Transm Infect 2009;85:111-5.

16. An Advisory Committee Statement (ACS), National Advisory Committee on Immunization (NACl). Updated recommendations on human papillomavirus (HPV) vaccines: 9-valent HPV vaccine and clarification of minimmum intervals between doses in the HPV immunization schedules. Ontario, Canada, 2016. http://www. healthycanadians.gc.ca/publications/healthy-living-vie-saine/humanpapillomavirus-9-valent-vaccine-update-recommendation-misesa-jour-recommandations-papillome-humain-vaccin-nonavalent/alt/ hpv-phv-eng.pdf (accessed 14 Feb 2017).

17. Szarewski A, Poppe WA, Skinner SR, et al. Efficacy of the human papillomavirus (HPV)-16/18 AS04-adjuvanted vaccine in women aged 15-25 years with and without serological evidence of previous exposure to HPV-16/18. Int J Cancer 2012;131:106-16.

18. Kjaer SK, Sigurdsson K, Iversen OE, et al. A pooled analysis of continued prophylactic efficacy of quadrivalent human papillomavirus (Types 6/11/16/18) vaccine against high-grade cervical and external genital lesions. Cancer Prev Res 2009;2:868-78.

19. Dillner J, Kjaer SK, Wheeler CM, et al. Four year efficacy of prophylactic human papillomavirus quadrivalent vaccine against low grade cervical, vulvar, and vaginal intraepithelial neoplasia and anogenital warts: randomised controlled trial. $B M J$ 2010;341:c3493.

20. Ferris D, Samakoses R, Block SL, et al. Long-term study of a quadrivalent human papillomavirus vaccine. Pediatrics 2014;134:e657-65.

21. Muñoz N, Kjaer SK, Sigurdsson K, et al. Impact of human papillomavirus (HPV)-6/11/16/18 vaccine on all HPVassociated genital diseases in young women. J Natl Cancer Inst 2010;102:325-39.

22. Stillo M, Carrillo Santisteve P, Lopalco PL. Safety of human papillomavirus vaccines: a review. Expert Opin Drug Saf 2015;14:697-712.

23. Ogilvie GS, Naus M, Money DM, et al. Reduction in cervical intraepithelial neoplasia in young women in British Columbia after introduction of the HPV vaccine: an ecological analysis. Int $J$ Cancer 2015;137:1931-7.

24. Canadian Immunization Committee. Recommendations for human papillomavirus immunization programs. Canada Communicable Disease Report 2014;40.

25. Shapiro GK, Guichon J, Prue G, et al. A multiple streams analysis of the decisions to fund gender-neutral HPV vaccination in Canada. Prev Med 2017;100:123-31.

26. Bonanni P, Bechini A, Donato $R$, et al. Human papilloma virus vaccination: impact and recommendations across the world. Ther Adv Vaccines 2015;3:3-12.

27. Marsh K, Chapman R, Baggaley RF, et al. Mind the gaps: what's missing from current economic evaluations of universal HPV vaccination? Vaccine 2014;32:3732-9. 
28. Patel C, Macartney K. Supporting the call for a gender-neutral human papillomavirus vaccination in Canada. CMAJ 2017;189:E118.

29. McClure CA, MacSwain MA, Morrison $\mathrm{H}$, et al. Human papillomavirus vaccine uptake in boys and girls in a school-based vaccine delivery program in Prince Edward Island, Canada. Vaccine 2015;33:1786-90.

30. Public Health Agency of Canada. Canada's provincial and territorial routine (and Catch-up) vaccination programs for infants and children 2017. http://www.phac-aspc.gc.ca/im/ptimprog-progimpt/table-1eng.php (accessed 8 May 2017).

31. Colbert Y. HPV vaccine for Nova Scotia boys called 'groundbreaking': CBC News, 2015. http://www.cbc.ca/news/canada/nova-scotia/ hpv-vaccine-for-nova-scotia-boys-called-groundbreaking-1.3031169 (accessed 07 May 2017).

32. Shapiro GK, Perez S, Rosberger Z. Including males in Canadian human papillomavirus vaccination programs: a policy analysis. CMAJ 2016;188:881-6.

33. Ghoussoub M. BC extends free HPV vaccinations to grade 6 boys. British Columbia, 2017. http://www.cbc.ca/news/canada/britishcolumbia/b-c-extends-free-hpv-vaccinations-to-grade-6-boys-1. 3925212

34. Cover JK, Nghi NQ, LaMontagne DS, et al. Acceptance patterns and decision-making for human papillomavirus vaccination among parents in Vietnam: an in-depth qualitative study post-vaccination. BMC Public Health 2012;12:629.

35. Fong K. Grade 6 boys to start receiving HPV vaccination in Saskatchewan 2017. 2017 http://globalnews.ca/news/3331430/ grade-6-boys-to-start-receiving-hpv-vaccinations-in-sask/ (accessed 08 May 2017).

36. Boone M. Boys too: HPV vaccination to be offered to all N.L. students in Grade 6. 2017 http://www.cbc.ca/news/canada/ newfoundland-labrador/hpv-vaccine-boys-newfoundland-1.4098941 (accessed 08 May 2017).

37. Drolet M, Bénard É, Boily MC, et al. Population-level impact and herd effects following human papillomavirus vaccination programmes: a systematic review and meta-analysis. Lancet Infect Dis 2015;15:565-80.

38. Ministère de la Santé et des Services Sociaux. Programme National de Santé Publique. 2008 http://publications.msss.gouv.qc.ca/ acrobat/f/documentation/2008/08-216-01.pdf

39. Bogaards JA, Wallinga J, Brakenhoff RH, et al. Direct benefit of vaccinating boys along with girls against oncogenic human papillomavirus: bayesian evidence synthesis. BMJ 2015;350:h2016.

40. Gilbert NL, Gilmour H, Dubé Ė, et al. Estimates and determinants of HPV non-vaccination and vaccine refusal in girls 12 to $14 \mathrm{y}$ of age in Canada: results from the Childhood National Immunization Coverage Survey, 2013. Hum Vaccin Immunother 2016;12:1484-90.

41. Perez S, Shapiro GK, Brown CA, et al. 'I didn't even know boys could get the vaccine': parents' reasons for human papillomavirus (HPV) vaccination decision making for their sons. Psychooncology 2015:24:1316-23.

42. Ogilvie GS, Remple VP, Marra F, et al. Parental intention to have daughters receive the human papillomavirus vaccine. CMAJ 2007;177:1506-12.

43. Allen JD, Coronado GD, Williams RS, et al. A systematic review of measures used in studies of human papillomavirus (HPV) vaccine acceptability. Vaccine 2010;28:4027-37.

44. Krawczyk AL, Perez S, Lau E, et al. Human papillomavirus vaccination intentions and uptake in college women. Health Psychol 2012;31:685-93.

45. Ogilvie GS, Remple VP, Marra F, et al. Intention of parents to have male children vaccinated with the human papillomavirus vaccine. Sex Transm Infect 2008;84:318-23.

46. Lee A, Ho M, Cheung CK, et al. Factors influencing adolescent girls' decision in initiation for human papillomavirus vaccination: a cross-sectional study in Hong Kong. BMC Public Health 2014:14:925

47. Zimet GD, Rosberger Z, Fisher WA, et al. Beliefs, behaviors and HPV vaccine: correcting the myths and the misinformation. Prev Med 2013:57:414-8

48. Hendry M, Lewis R, Clements A, et al. 'HPV? Never heard of it!': a systematic review of girls' and parents' information needs, views and preferences about human papillomavirus vaccination. Vaccine 2013;31:5152-67.

49. Holman DM, Benard V, Roland KB, et al. Barriers to human papillomavirus vaccination among US adolescents: a systematic review of the literature. JAMA Pediatr 2014;168:76-82.

50. Patel PR, Berenson AB. Sources of HPV vaccine hesitancy in parents. Hum Vaccin Immunother 2013;9:2649-53.

51. Walhart T, Parents WT. Parents, adolescents, children and the human papillomavirus vaccine: a review. Int Nurs Rev 2012;59:305-11.
52. Krawczyk A, Knäuper B, Gilca V, et al. Parents' decision-making about the human papillomavirus vaccine for their daughters: I. Quantitative results. Hum Vaccin Immunother 2015;11:322-9.

53. Trim K, Nagji N, Elit L, et al. Parental knowledge, attitudes, and behaviours towards human papillomavirus vaccination for their children: a systematic review from 2001 to 2011. Obstet Gynecol Int 2012;2012:1-12

54. Gowda C, Dempsey AF. The rise (and fall?) of parental vaccine hesitancy. Hum Vaccin Immunother 2013;9:1755-62.

55. Garcini LM, Galvan T, Barnack-Tavlaris JL. The study of human papillomavirus (HPV) vaccine uptake from a parental perspective: a systematic review of observational studies in the United States. Vaccine 2012;30:4588-95.

56. Larson HJ, Jarrett C, Eckersberger E, et al. Understanding vaccine hesitancy around vaccines and vaccination from a global perspective: a systematic review of published literature, 2007-2012. Vaccine 2014;32:2150-9.

57. Forster AS, Rockliffe L, Chorley AJ, et al. A qualitative systematic review of factors influencing parents' vaccination decision-making in the United Kingdom. SSM Popul Health 2016;2:603-12.

58. Perez S, Tatar O, Gilca V, et al. Untangling the psychosocial predictors of HPV vaccination decision-making among parents of boys. Vaccine 2017;35:4713-21.

59. Lindley MC, Jeyarajah J, Yankey D, et al. Comparing human papillomavirus vaccine knowledge and intentions among parents of boys and girls. Hum Vaccin Immunother 2016;12:1519-27.

60. Radisic G, Chapman J, Flight I, et al. Factors associated with parents' attitudes to the HPV vaccination of their adolescent sons: a systematic review. Prev Med 2017;95:26-37.

61. Government of Canada. Immunization coverage and registries. 2016 https://www.canada.ca/en/public-health/services/immunizationcoverage-registries.html\#a3 (accessed 07 Feb 2017).

62. Champion VL, Skinner CS. The health belief model. In: Glanz $\mathrm{K}$, Rimer BK, Viswanath KE, eds. Health behavior and health education: theory, research, and practice. 4th ed: John Wiley \& Sons, 2008:45-65.

63. Brewer NT, Fazekas KI. Predictors of HPV vaccine acceptability: a theory-informed, systematic review. Prev Med 2007;45:107-14.

64. Cunningham MS, Davison C, Aronson KJ. HPV vaccine acceptability in Africa: a systematic review. Prev Med 2014;69:274-9.

65. Prue G, Santin O. HPV vaccine acceptance in male adolescents. Psychooncology 2015;24:1327-9.

66. Perez S, Tatar O, Shapiro GK, et al. Psychosocial determinants of parental human papillomavirus (HPV) vaccine decision-making for sons: methodological challenges and initial results of a panCanadian longitudinal study. BMC Public Health 2016;16:1223.

67. Weinstein ND. The precaution adoption process. Health Psychol 1988; 7:355-86.

68. Court of Appeal of Alberta. J.S.C. v. Wren, 1986 ABCA 249 (CanLII) 31-12-1986. http://www.canlii.org/en/ab/abca/doc/1986/ 1986abca249/1986abca249.html

69. Osborne JW. Best practices in data cleaning: a complete guide to everything you need to do before and after collecting your data: Sage Publications, 2012

70. Shapiro GK, Holding A, Perez S, et al. Validation of the vaccine conspiracy beliefs scale. Papillomavirus Res 2016;2:167-72.

71. Perez S, Tatar O, Ostini R, et al. Extending and validating a human papillomavirus (HPV) knowledge measure in a national sample of Canadian parents of boys. Prev Med 2016;91:43-9.

72. Waller J, Ostini R, Marlow LA, et al. Validation of a measure of knowledge about human papillomavirus (HPV) using item response theory and classical test theory. Prev Med 2013;56:35-40.

73. McRee AL, Brewer NT, Reiter PL, et al. The Carolina HPV immunization attitudes and beliefs scale (CHIAS): scale development and associations with intentions to vaccinate. Sex Transm Dis 2010;37:234-9.

74. Larson HJ, Jarrett C, Schulz WS, et al. Measuring vaccine hesitancy: the development of a survey tool. Vaccine 2015;33:4165-75.

75. Perez S, Shapiro GK, Tatar O, et al. Development and validation of the human papillomavirus attitudes and beliefs scale in a National Canadian sample. Sex Transm Dis 2016;43:626-32.

76. DeSimone JA, Harms PD, DeSimone AJ. Best practice recommendations for data screening. J Organ Behav 2015;36:171-81.

77. LaClair BJ, Smith S, Woodward J. Attitudes and concerns of Kansas parents related to childhood immunization. Immunize Kansas Kids 2014.

78. Meade AW, Craig SB. Identifying careless responses in survey data. Psychol Methods 2012;17:437-55. 